\title{
Was determiniert Einstellungen zu den Folgen der Aufnahme von Flüchtlingen in Deutschland? Ergebnisse auf Basis des GESIS-Panels 2016
}

\author{
Peter Reinhardt $\mathbb{D} \cdot$ Cornelia Frings
}

Online publiziert: 1. Juli 2020

(C) Der/die Autor(en) 2020

Zusammenfassung Die sogenannte Flüchtlingskrise ist eines der dominierenden Themen in Westeuropa und steht im Zusammenhang mit zunehmend erfolgreichen rechtspopulistischen Parteien und Bewegungen. Die deutsche Gesellschaft scheint gespalten zu sein in der Frage, ob die Aufnahme von Flüchtlingen für Deutschland mehr Chancen oder mehr Risiken mit sich bringt. Dieser Artikel analysiert die Frage nach den Gründen für eine optimistische bzw. pessimistische Sicht auf die Folgen der Flüchtlingsaufnahme. Anknüpfend an drei zentrale Erklärungsansätze der vergleichenden Forschung zu immigrationsbezogenen Einstellungen testen wir den Einfluss von sozioökonomischen Determinanten, kulturellen Determinanten (Ethnozentrismus und soziales Vertrauen) sowie von Kontakt mit Flüchtlingen. Datengrundlage bildet das GESIS-Panel 2016. Soziales Vertrauen, Ethnozentrismus und Kontakt erweisen sich als erklärungskräftige Determinanten. Insbesondere Ethnozentrismus hat einen starken Effekt auf die Einstellungen zu den Konsequenzen der Aufnahme von Flüchtlingen in Deutschland. Soziales Vertrauen wirkt offenbar weniger direkt, sondern eher indirekt, vermittelt über Ethnozentrismus.

Schlüsselwörter Soziales Vertrauen · Ethnozentrismus · Kontakt · Immigration · Migrationsskepsis

\footnotetext{
Zusatzmaterial online Zusätzliche Informationen sind in der Online-Version dieses Artikels (https://doi.org/10.1007/s11615-020-00254-2) enthalten. Tab. 1: Liste der verwendeten Items (*Skala umgedreht). Tab. 2: Effekte der Modelle 1-4, standardisierte Koeffizienten aus den linearen Strukturgleichungsmodellen mit Full-Information Maximum Likelihood Methode. Tab. 3: Direkte und indirekte Effekte aus Modell 5, standardisierte Koeffizienten aus dem linearem Strukturgleichungsmodelle mit Full-Information Maximum Likelihood Methode.

P. Reinhardt $(\square) \cdot$ C. Frings

Institut für Politikwissenschaft, Bereich „Politik und Wirtschaft“, Johannes Gutenberg-Universität Mainz, Jakob-Welder-Weg 12, 55128 Mainz, Deutschland

E-Mail: reinhardt@politik.uni-mainz.de
} 


\title{
What Determines Attitudes Toward the Consequences of the Reception of Refugees in Germany? Results from the GESIS Panel 2016
}

\begin{abstract}
The so-called "refugee crisis" is one of the dominating issues in Western Europe and is associated with increasingly successful right-wing populist parties and movements. Moreover, the German population seems to be divided on whether receiving refugees brings more opportunities or more risks for Germany. This article analyzes the underlying reasons for an optimistic or pessimistic view of the consequences of incoming refugees. Following the three prominent theoretical perspectives in the comparative research on anti-immigrant attitudes, we test the influence of socio-economic determinants, cultural determinants (ethnocentrism and social trust) as well as the influence of contact with refugees. The basis for the data is the GESIS Panel 2016. Social trust, ethnocentrism, and contact seem to be key predictors. In particular, ethnocentrism has a strong effect on attitudes toward the consequences of accepting refugees into Germany. Social trust apparently has a less direct, or rather an indirect effect, mediated by ethnocentrism.
\end{abstract}

Keywords Social trust $\cdot$ Ethnocentrism $\cdot$ Contact $\cdot$ Immigration $\cdot$ Migration scepticism

\section{Einführung und Fragestellungen}

Die als „Flüchtlingskrise“ bezeichnete Zunahme der nach Deutschland einreisenden Flüchtlinge und anderer Migranten zwischen 2015 und 2016 hat in Deutschland, wie auch in den anderen europäischen Staaten, zu einer deutlichen Intensivierung der politischen und gesellschaftlichen Auseinandersetzung mit dem Thema Zuwanderung geführt. Der deutliche Rückgang der Zahl der Flüchtlinge seit 2016 hat diese Debatten nicht zum Erliegen gebracht. Fragen der Integration und der Auswirkungen dieser Zuwanderung für die Aufnahmegesellschaft werden kontrovers und teilweise emotional sehr aufgeladen diskutiert und überlagern zum Teil andere politische Themen (Pickel et al. 2018).

Es geht in diesen Diskursen aber auch um die weitaus grundlegendere Frage der zukünftigen Ausrichtung der deutschen wie europäischen Einwanderungs- und Flüchtlingspolitik, in der zentrale Streitfragen nach wie vor ungelöst sind. Die Haltungen der Bevölkerung zu dieser Frage erscheinen gespalten (Schmidt und Weick 2017, S. 4; Pickel und Pickel 2019; Eisnecker und Schupp 2016): Einerseits gibt es viele zivilgesellschaftliche Organisationen und ehrenamtliche Helfer, die sich seit 2015 darum bemühen, die neu in Deutschland Angekommenen bei der Integration in die Gesellschaft zu unterstützen, und dies als gesamtgesellschaftliche Aufgabe begreifen. Der Aufnahme von Flüchtlingen und der damit einhergehenden Zunahme an ethnischer und kultureller Diversität begegnen aber andererseits auch viele Bürger offenbar mit Skepsis, Sorge und Ablehnung. Dies zeigen beispielsweise die Wahlerfolge der rechtspopulistischen Alternative für Deutschland (AfD). Ursprünglich in der Eurokrise als Single-Issue-Partei angetreten, profilierte sich die Partei im Zuge der „Flüchtlingskrise“ von 2015 als Antizuwanderungspartei (Schmitt-Beck et al. 
2017, S. 275). Für viele deutsche Wähler schien sie mit diesem programmatischen Schwerpunkt als Wahlalternative zu den etablierten Parteien offenbar attraktiv. Wie aktuelle empirische Studien belegen, scheint sich die AfD primär zu einem Sammelbecken für Personen entwickelt zu haben, die mit der Flüchtlingspolitik der Bundesregierung nicht einverstanden sind (Hambauer und Mays 2018; Lengfeld und Dilger 2018). Mit einer Nulltoleranzposition in Fragen der Asyl- und Migrationspolitik gelang es der AfD, sich in den meisten Landesparlamenten zu etablieren, und sie ist seit 2017 als drittstärkste Kraft im Bundestag vertreten. Es gibt zudem Hinweise darauf, dass auch unter den Wählern anderer Parteien eine einwanderungsskeptische Haltung offenbar weit verbreitet sein könnte. Während sich $96 \%$ der im Rahmen des German Longitudinal Election Panel (GLES) befragten AfD-Wähler dafür aussprachen, dass Deutschland weniger Flüchtlinge aufnehmen solle, stimmten $56 \%$ der Wähler anderer Parteien ebenfalls dieser Aussage zu (Hambauer und Mays 2018, S. 142). Im vorliegenden Beitrag möchten wir untersuchen, wie sich diese Varianz in der Bewertung der Chancen und Risiken dieser Form von Immigration erklären lässt. Unser Fokus liegt hierbei auf der Beurteilung der Folgen für die deutsche Gesellschaft. Was bedingt, ob die Aufnahme von Flüchtlingen eher optimistisch im Sinne einer gesellschaftlichen Chance interpretiert wird oder ob eine eher skeptische Haltung eingenommen wird? Um dies zu untersuchen, testen wir eine Reihe von Erklärungsfaktoren, die in der Literatur diskutiert werden. Vor allem interessiert uns, welchen Erklärungsbeitrag kulturelle Determinanten, wie das generelle soziale Vertrauen in andere Menschen und die Neigung zu Ethnozentrismus, bei der Erklärung dieser Varianz in der Beurteilung von Flüchtlingen leisten können. Darüber hinaus überprüfen wir, inwiefern sozioökonomische Determinanten und sozialer Kontakt mit Geflüchteten Einfluss nehmen.

\section{Aktueller Forschungsstand}

Die Erforschung von Einstellungen zu Immigranten und zur Immigration ganz allgemein hat in der vergleichenden politikwissenschaftlichen Forschung wie auch in der vergleichenden Soziologie eine lange Tradition ${ }^{1}$. Ein Großteil der vorliegenden empirischen Studien konzentriert sich auf Einstellungen der aufnehmenden, einheimischen Bevölkerung, wobei ein Teil der Studien auf Einstellungen zu den Immigranten als abhängige Variable ausgerichtet ist, während ein anderer Teil Einstellungen zur Immigration als solche ins Zentrum stellt. Länderschwerpunkte bilden die USA und Westeuropa, wobei einige Studien älteren Datums auch Deutschland miteinbeziehen (Fetzer 2000; Gorodzeisky und Semyonov 2019; Lahav 2004; Louis et al. 2007; McLaren 2003; Citrin und Sides 2008; Davidov et al. 2008; Mughan und Paxton 2006; Meuleman et al. 2009) oder sich nur auf den Einzelfall Deutschland konzentrieren (Semyonov et al. 2004).

Aktuelle politikwissenschaftliche Beiträge, die Einstellungen zu Migranten allgemein sowie Flüchtlingen im Besonderen und deren Bestimmungsfaktoren bezo-

\footnotetext{
${ }^{1}$ Für einen Überblick über den politikwissenschaftlichen Forschungsstand siehe Hainmüller und Hopkins (2014); der soziologische Forschungsstand wird in Ceobanu und Escandell (2010) zusammengefasst.
} 
gen auf Deutschland differenziert und theoriegeleitet untersuchen, gibt es hingegen bisher kaum². Die vorliegenden Forschungsarbeiten, die die Zeit nach der vermehrten Aufnahme von Flüchtlingen 2015 beleuchten, kommen überwiegend aus der Soziologie. Sie untersuchen beispielsweise die aktuelle Stimmung in der Bevölkerung bezüglich der Aufnahme von Flüchtlingen, die Übereinstimmung zwischen der geltenden Rechtslage und den Einstellungen der Bevölkerung oder die Frage, von welchen migrantenbezogenen Merkmalen die Akzeptanz der Ausländer in der deutschen Bevölkerung abhängt (Gerhards et al. 2016; Bertelsmann Stiftung 2017; SVR 2018; Czymara und Schmidt-Catran 2016). Einige Autoren konzentrieren sich auch auf einzelne Bestimmungsfaktoren, wie zum Beispiel Weber, der den komplexen Zusammenhang zwischen Zuwandererzahl, Kontakteffekten und Skepsis gegenüber Immigranten analysiert (Weber 2016). Die politikwissenschaftliche Forschung setzt derzeit eher andere Schwerpunkte. So ist einerseits das Erstarken des Rechtspopulismus in Folge der Flüchtlingskrise von Seiten der politikwissenschaftlichen Forschung mit Interesse und Aufmerksamkeit begleitet worden. Die vorliegenden Publikationen aus dem Bereich der Rechtsextremismus- bzw. Rechtspopulismusforschung beschäftigen sich einerseits mit der Angebotsseite des politischen Markts und untersuchen die Entstehung der AfD, ihre programmatische Veränderung seit ihren Anfängen als Partei sowie ihre ideologische Verortung (Arzheimer 2015, 2017; Decker 2016; Lewandowsky et al. 2016; Mudde 2017). Auch mit der PEGIDA-Bewegung als außerparlamentarische Sammelbewegung haben sich einige Vertreter aus den Reihen der Demokratie- und Protest- sowie der Migrationsforschung beschäftigt (Rucht et al. 2015; Geiges et al. 2015; Schammann 2015; Decker 2015). Relativ viel Interesse hat auch die Nachfrageseite des politischen Markts und damit besonders das Wählerklientel der AfD erfahren (Lengfeld und Dilger 2018; Schmitt-Beck et al. 2017; Hambacher und Mays 2018; Decker 2016; Rippl und Seipel 2018).

Dass aktuelle politikwissenschaftliche Beiträge selten sind, die Einstellungen zu Flüchtlingen ins Zentrum stellen, ist insofern eine relevante Forschungslücke, weil es wesentlich von den Wahrnehmungen und Haltungen gesellschaftlicher Mehrheiten in den Aufnahmeländern abhängt, wie Immigration gesellschaftlich bewältigt wird und wie sie sich auf die Politik in den Aufnahmeländern auswirkt (Hainmueller und Hopkins 2014, S. 226). Ähnlich argumentieren auch Pickel und Pickel (2018) in einem aktuellen Beitrag mit dem provokanten Titel „Migration als Gefahr für die politische Kultur?" Sie vertreten darin die Auffassung, dass die Integrationsbereitschaft der Mitglieder der Aufnahmegesellschaft neben der Integrationsbereitschaft der Migranten für eine erfolgreiche Integration entscheidend ist. So seien es nicht die durch die jüngste Fluchtbewegung in Deutschland wie in einigen anderen europäischen Ländern ausgelösten soziostrukturellen Veränderungen, beschrieben als ethnischer, kultureller und religiöser Pluralisierungsschub für die Aufnahmegesellschaften, die der Flüchtlingskrise ihre öffentlich zugewiesene Einschätzung als Krise eingebracht hätten. Vor allem Verweise auf die Probleme, die diese Bewegungen für die gesellschaftliche Integration, den gesellschaftlichen Zusammenhalt und die

\footnotetext{
2 Zu den Ausnahmen aus politikwissenschaftlicher Sicht zählt ein Beitrag von Schmidt und Weick (2017), der auf Basis von GESIS-Paneldaten von 2016 Einstellungen zu Ausländern untersucht und diverse demografische sowie sozialpsychologische Konstrukte als Bestimmungsfaktoren miteinbezieht.
} 
Inklusion von sozialen Gruppen mit sich brächten, und damit die Bevölkerungsmeinungen zu diesen gesellschaftlichen Entwicklungen seien entscheidend (Pickel und Pickel 2018, S. 301). Die Autoren verweisen zudem auf mögliche Wechselwirkungen zwischen der durch die Bürger der Aufnahmegesellschaft subjektiv wahrgenommenen Integrationsfähigkeit einer Gesellschaft und dem Zusammenhalt der politischen Gemeinschaft sowie der Akzeptanz der Demokratie als Regimeform (Pickel und Pickel 2018, S. 298). Pickel und Pickel (2018) skizzieren damit ein neues und bisher wenig bearbeitetes Forschungsprogramm für die vergleichende politische Kulturforschung, mit „,noch [...] (erheblichem) Bedarf an politischen Studien“ (Pickel et al. 2018, S. 6) ${ }^{3}$. Einstellungsbezogene Studien stellten zudem eine wesentliche Ergänzung zur policyanalytischen Perspektive auf das Thema Migration innerhalb der Politikwissenschaft dar: ,[...] eine reine auf die Migrationsströme gerichtete, PolicyMaßnahmen analysierende Vorgehensweise [greift] nur einen Aspekt auf, und wie es scheint nicht den für die Integration und Inklusion der Gesellschaft wichtigsten. Die derzeit massivsten Wirkungen von Migration finden sich in den Bevölkerungsmeinungen. Dort bündeln sich Positionierungen, die auf der Ebene der Kultur angesiedelt sind“ (Pickel und Pickel 2018, S. 302). Gleichzeitig können differenziertere Analysen der Einstellungen zu Migranten und ihrer Ursachen aktuelle policyanalytische Arbeiten zum Themenfeld der Migrationspolitik wesentlich bereichern. Die von Gary Freeman (1995) formulierte Hypothese eines sogenannten ,opinion-policy gap“, wonach Migrationspolitik in liberalen Demokratien durch einen persistenten Gegensatz zwischen tendenziell liberalen ,policies“ und deutlich restriktiveren Einstellungen der Bevölkerung gekennzeichnet sei, dominiert dort derart, dass sie sogar schon als Gesetz der Migrationsforschung bezeichnet wurde (Schammann 2015, S. 310). Es gibt aber nur wenige empirische Studien, die bezogen auf den deutschen Kontext Einstellungen zu immigrationsbezogenen Fragestellungen und ihre Bestimmungsfaktoren empirisch untersuchen (Fietkau 2016).

\section{Hypothesen}

In der Forschungsdebatte um die Ursachen von Einstellungen zu Immigranten und deren Integration in die Aufnahmegesellschaften lassen sich drei zentrale Argumentationslinien ausmachen (Ceobanu und Escandell 2010, S. 317): ein ökonomischer Erklärungsansatz, der sozioökonomische Faktoren als Determinanten hervorhebt, ein kultureller Erklärungsansatz, der tief in der Persönlichkeit verankerte generelle Einstellungen zu anderen Menschen und zur eigenen Gruppenzugehörigkeit als zentrale identitätsstiftende Erklärungsfaktoren betont, sowie ein dritter Ansatz, der Kontakt und soziale Interaktion als entscheidende Ursachen benennt.

Aus Perspektive des ökonomischen Erklärungsansatzes sind Einstellungen zur Integration von Flüchtlingen und deren Konsequenzen vor allem von der individuellen sozioökonomischen Ressourcenausstattung und den damit einhergehenden, individuellen Fähigkeiten abhängig (Malchov-Møller et al. 2009; Scheve und Slaughter

\footnotetext{
3 Erste Beiträge zu diesem Forschungsprogramm finden sich in dem dazugehörigen Sonderband der Zeitschrift für Vergleichende Politikwissenschaft von 2018 (vgl. Pickel et al. 2018).
} 
2001; Mayda 2004). Begründet wird diese Annahme unter anderem damit, dass sich Menschen, die über ein geringes Maß an Humankapital und ein geringes Einkommen verfügen, eher durch Migranten in ihrem sozioökonomischen Status bedroht sehen und ökonomische Deprivation, Wohlstands- oder Arbeitsplatzverluste befürchten ${ }^{4}$. Bildung und Einkommen sind aus dieser Perspektive betrachtet materielle Ressourcen und ablehnende Haltungen gegenüber Flüchtlingen basieren entsprechend auf ökonomischen Kalkulationen.

Empirische Studien weisen aber darauf hin, dass diese wahrgenommene ökonomische Bedrohung möglicherweise gar nicht so entscheidend ist und gerade Bildung nicht nur als materielle Ressource auf Einstellungen zu Flüchtlingen wirken könnte. Vielmehr gibt es Hinweise darauf, dass Menschen, die höher gebildet sind, generell optimistischer, toleranter und liberaler hinsichtlich Zuwanderung und der Beurteilung ihrer Auswirkungen eingestellt sind (vgl. Hainmüller und Hiscox 2007; Schweitzer et al. 2005, S. 171; Margaryan et al. 2018). Anknüpfend an die frühen Überlegungen von Inglehart (1970) lässt sich das damit begründen, dass Bildung zu einer kognitiven Mobilisierung beitragen kann. Das heißt, mit dem Grad der formalen Bildung steigt zugleich die Fähigkeit und Bereitschaft, neue Informationen und neues Wissen aufzunehmen und zu verarbeiten und neu eingetretene Situationen differenzierter und offener zu beurteilen, was sich auch auf die Haltung zu den Auswirkungen der Integration von Flüchtlingen auswirken könnte. Das führt uns zu einer ersten Hypothese:

H1: Bildung und Einkommen haben einen positiven Effekt auf die Einschätzung der wahrgenommenen Folgen der Integration von Flüchtlingen.

Kulturelle Erklärungsansätze gehen davon aus, dass die Haltung zu Immigranten und zur Immigration in erster Linie durch tief in der Persönlichkeit verankerte, längerfristig stabile, individuelle Einstellungen beeinflusst wird (Berg 2015). Das dabei unterstellte kausale Verhältnis zwischen solchen generellen Einstellungen und spezifischen Haltungen und Überzeugungen wird dabei allerdings selten genauer erläutert und begründet. Hilfreich ist in diesem Zusammenhang ein Rekurs auf die sozialpsychologische Einstellungsforschung, die sich seit vielen Jahren, insbesondere im Rahmen der sogenannten Einstellungs-Verhaltens-Kontroverse, mit unterschiedlichen Einstellungskategorien und ihren Relationen beschäftigt hat (Ajzen und Fishbein 1980, 2005) . Diese unterscheidet zwischen generellen und spezifischen Einstellungen. Generelle Einstellungen beziehen sich demnach auf eine breite Kategorie von Einstellungsobjekten (z. B. alle Menschen) und haben chronisch zu-

\footnotetext{
${ }^{4}$ Wie auch aktuelle Forschungsbeiträge zu den Determinanten rechtspopulistischer Orientierungen vermuten, könnte die Zunahme dieses wahrgenommenen Konkurrenzdrucks eine Begleiterscheinung von Globalisierungsprozessen und damit einhergehenden Modernisierungsschüben sein. Diese hätten neue wirtschaftliche Unsicherheiten und eine Prekarisierung von Arbeitsverhältnissen mit sich gebracht, von denen Menschen mit geringem Bildungsgrad und geringem Einkommen am stärksten betroffen seien (Lengfeld 2017, S. 211; Rippl und Seipel 2018, S. 239).

${ }^{5}$ Einstellungen sind demnach psychologische Tendenzen, die darin zum Ausdruck kommen, dass ein bestimmtes Einstellungsobjekt (z. B. eine Gruppe von Personen oder ein bestimmter Sachverhalt) bewertet wird (vgl. Eagly und Chaiken 1993, S. 1).
} 
gängliche Überzeugungen als Grundlage (Ajzen 2001, S. 35). Sie werden in Sozialisationsprozessen erworben und sind über Zeit und Kontext hinweg relativ stabil. Spezifische Einstellungen sind im Unterschied dazu auf enger definierte, spezifische Einstellungsobjekte ausgerichtet und basieren auf einem Set an spezifischen Überzeugungen, die in Abhängigkeit von situativ gegebenen Bedingungen, beziehungsweise der Wahrnehmung dieser Bedingungen durch den Akteur, neu gebildet beziehungsweise aktiviert werden müssen. Entsprechend sind spezifische Einstellungen weniger stabil als generelle Einstellungen und können eher in Abhängigkeit von den jeweiligen situativen Gegebenheiten variieren. Sie werden aber nach den Annahmen der kognitiven Sozialpsychologie nicht nur von der Wahrnehmung und Einschätzung situationsspezifischer Merkmale, sondern auch von generellen Einstellungen beeinflusst. Diese fungieren in komplexen Entscheidungssituationen als generelle Bezugsrahmen, Bewertungsmaßstäbe und Interpretationshilfen, die bei der Beurteilung von spezifischen Einstellungsobjekten herangezogen werden können. Generelle Einstellungen stellen damit eine Art fest verankerte Struktur im Gedächtnis dar, die in allen Phasen der Verarbeitung von Informationen über aktuelle politische Entwicklungen und deren Beurteilung herangezogen werden kann (Houston und Fazio 1989; Eagly und Chaiken 1993; Markus und Zajonc 1985; ausführlich Frings 2010, S. 162). Wir argumentieren im Folgenden, dass vor allem zwei kulturelle Erklärungsfaktoren, die in der Literatur zu immigrationsspezifischen Einstellungen diskutiert werden, als solche generellen Bezugsrahmen die spezifische Beurteilung der aktuellen Integration von Flüchtlingen beeinflussen könnten: zum einen soziales Vertrauen und zum anderen Ethnozentrismus.

Nach den Annahmen des in der politikwissenschaftlichen Vertrauens- und Sozialkapitalforschung dominierenden soziologischen Vertrauensansatzes ist das generelle Vertrauen in andere Menschen eine generelle Einstellung, die im Rahmen von primärer und sekundärer Sozialisation erworben und dann auf Menschen ganz allgemein, unabhängig von deren Herkunft, Hautfarbe, ethnischer oder religiöser Zugehörigkeit, übertragen wird. Soziales Vertrauen gilt seit den Anfängen der politischen Kulturforschung als wesentliche kulturelle Grundlage für gesellschaftlichen Zusammenhalt sowie stabile und leistungsfähige Demokratien und Volkswirtschaften. Im Zuge der intensiven Diskussionen um Sozialkapital und seine positiven Effekte sind diese Überlegungen weiter ausgearbeitet und erstmals auch systematisch empirisch überprüft worden (Frings 2010, S. 36; Putnam et al. 1993; Paxton 2002; Zmerli und Newton 2008). Diese unterstellten Makrozusammenhänge fußen auf der mikrotheoretischen Annahme, dass Menschen, die ein hohes Maß an sozialem Vertrauen in den generalisierten Anderen internalisiert haben, generell kooperationsbereiter, toleranter, engagierter und eher willens sind, auf ihnen unbekannte Menschen zuzugehen. Dies gilt insbesondere für solche Menschen, die nicht dem eigenen vertrauten Umfeld entstammen oder einen anderen ethnischen, kulturellen, religiösen oder sozialen Hintergrund haben (van der Linden et al. 2017, S. 2). Menschen mit einem hohen $\mathrm{Maß}$ an sozialem Vertrauen verfügen damit über die notwendigen ,civic skills“, die gesellschaftliche Integration in zunehmend multikulturell geprägten Gesellschaften erleichtern (Berning und Ziller 2017). Rustenbach (2010, S. 57) nimmt beispielsweise an: ,higher trust should also be related to not blaming immigrants unless one has had a personal negative experience with them. That is, some natives blame many 
societal problems on immigrants, such as high crime rates or unemployment, and this should occur less for individuals with high trust levels“. Dennoch gibt es bislang relativ wenige empirische Studien, die Vertrauen als kulturelle Variable miteinbezogen und mögliche positive Effekte auf migrationsbezogene Einstellungen sowie ihre Wechselwirkung mit anderen Erklärungsfaktoren in den Blick genommen haben (zu den weiteren Ausnahmen gehören Citrin und Sides 2008; Crepaz et al. 2014; Herreros und Criado 2009; van der Linden et al. 2017). Vor diesem Hintergrund möchten wir in unserem Beitrag die folgende Hypothese testen:

H2: Soziales Vertrauen hat einen positiven Effekt auf die Einschätzung der wahrgenommenen Folgen der Integration von Flüchtlingen.

Zahlreicher sind die Beiträge zum kulturellen Erklärungsansatz, die gruppenbezogene Einstellungen als Determinanten von immigrationsbezogenen Einstellungen untersuchen und dabei an die theoretischen Annahmen der „social identity theory“ von Tajfel et al. (1979) anknüpfen (Ceobanu und Escandell 2010; Sides und Citrin 2007; Hainmüller und Hopkins 2014; Banks 2016). Aus dieser Perspektive konstituieren sich soziale Gemeinschaften oder Gruppen unter anderem durch Grenzziehungen (Fuchs et al. 1993). Demnach neigen Menschen dazu, sich und andere anhand bestimmter Gemeinsamkeiten einer Ingroup zuzuordnen und gleichzeitig diese durch Markierung von Unterschieden von einer Outgroup abzugrenzen, wodurch eine ,Wir-vs.-die Anderen“-Kategorisierung erzeugt wird (Banks 2016, S. 637). Wie Tajfel et al. (1979) herausstellen, resultiert dieses Bedürfnis aus dem Streben nach einer positiven sozialen Identität, welche aus der Aufwertung der eigenen Gruppe und der Abwertung der Fremdgruppe gespeist wird (Weins 2004, S. 86f). Dabei wird die Eigengruppe häufig über die Zugehörigkeit zu einer bestimmten als homogen wahrgenommenen Kultur, Ethnie oder Nation gebildet; Abgrenzungen erfolgen über kulturelle Merkmale wie Religion, Sprache oder Ethnie und werden als latente Bedrohung für diese Homogenität wahrgenommen. Diese auf Gruppenstereotypen basierenden Einstellungen, die die Eigengruppe idealisieren und die Fremdgruppe abwerten, werden in der politischen Soziologie häufig unter dem Begriff des Ethnozentrismus subsumiert (Fuchs et al. 1993; Heyder und Schmidt 2003), der uns auch für unsere Untersuchung am geeignetsten erscheint. Da die soziale Identität etwas sehr Grundlegendes ist, ist auch hier anzunehmen, dass sich Ethnozentrismus aus tief in der Persönlichkeit verankerten generellen Einstellungen speist, die spezifischen Einstellungen bezüglich der Chancen und Risiken der Integration von Flüchtlingen kausal vorgelagert sein sollten.

Dass der generelle Hang zu ethnozentrischem Denken ein erklärungskräftiger Bestimmungsfaktor von Einstellungen zu Immigranten und zur Immigration ist, ist insbesondere in empirischen Studien zur USA verschiedentlich getestet und empirisch bestätigt worden (Haubert und Fussell 2006; Kinder und Kam 2009; Valentino et al. 2013; Banks 2016). Es gibt zudem empirische Belege dafür, dass die wahrgenommene kulturelle Affinität zur Outgroup, die sich an Merkmalen wie eigenem Migrationshintergrund oder eigener Zugehörigkeit zu einer religiösen Minderheit festmachen kann, Einstellungen zu Immigranten positiv beeinflussen kann (Zamora-Kapoor et al. 2013, S. 306, Marfouk 2018). Gleichzeitig warnen Autoren wie 
Rydgren (2008) eindringlich davor, einfach a priori anzunehmen, dass skeptische Haltungen gegenüber Immigranten zwangsläufig Ausdruck einer ethnozentrischen, fremdenfeindlichen oder sogar rassistischen generellen Einstellung seien. Denkbar wäre auch, dass eine kritische Sichtweise aus ganz anderen, konkreten Erfahrungen mit den Problemen und Hürden der gesellschaftlichen Integration und der daraus resultierenden Sorge einer Überlastung des Gemeinwesens resultiert und völlig unabhängig von genereller Fremdenfeindlichkeit ist. Rydgren (2008, S. 738) plädiert entsprechend dafür, es als eine konsequent empirische Frage zu betrachten, wie sehr Fremdenfeindlichkeit und Ethnozentrismus Immigrationsskepsis beeinflussen. Vor diesem Hintergrund erscheinen auch Operationalisierungsansätze wenig sinnvoll, die Ethnozentrismus selbst über das Vorliegen von negativen Einstellungen gegenüber Immigration messen (Hooghe et al. 2013). Diesem Plädoyer folgend, möchten wir untersuchen, wie stark spezifische Einstellungen zu den Folgen der Zuwanderung für die Gesellschaft tatsächlich von tiefer liegenden ethnozentrischen Einstellungen als generellem Bezugsrahmen beeinflusst werden.

H3: Ethnozentrismus hat einen negativen Effekt auf die Einschätzung der wahrgenommenen Folgen der Integration von Flüchtlingen.

Ein dritter Erklärungsansatz der Immigrationsforschung sieht im Kontakt zu Zugewanderten eine wesentliche Ursache für immigrationsbezogene Einstellungen. Dieser Kontakt schaffe Möglichkeiten zur sozialen Interaktion und zu interkulturellen Begegnungen. Auf diese Weise reduzierten sich Fehlwahrnehmungen und Vorurteile und dies führe als Folge zu positiverer Einstellung gegenüber Immigranten und deren Integration in die Aufnahmegesellschaft (Berg 2015, S. 28; Freitag und Rapp 2013; Pettigrew et al. 2011). Empirische Befunde aus Deutschland, die zeigen, dass die Skepsis gegenüber Zuwanderung vor allem in den Regionen überrepräsentiert ist, in denen der Migrantenanteil gering ist, scheinen diese Annahme zu plausibilisieren (Weber 2016). Eine empirische Studie von Schmidt und Weick (2017), die die Einstellungen der deutschen Bevölkerung zu Zuwanderern von 1980 bis 2016 untersucht, belegt zudem, dass Kontakt die Einstellung zu Migranten determiniert. Eine vierte Hypothese, die wir testen, lautet entsprechend:

H4: Kontakt hat einen positiven Effekt auf die Einschätzung der wahrgenommenen Folgen der Integration von Flüchtlingen.

\section{Daten, Operationalisierung und methodische Vorgehensweise}

Datengrundlage unserer Analysen bildet das GESIS-Panel (ZA5665, Version 23-0$0)$. Es handelt sich hierbei um ein probabilistisches Mixed-mode-Design, in dessen Rahmen die Panelisten seit Februar 2014 alle zwei Monate sowohl online als auch schriftlich befragt werden. Die ursprüngliche Stichprobe enthielt ca. 4900 Teilnehmer, wobei 2016 eine Auffrischungsstichprobe aus dem ALLBUS (Allgemeine Bevölkerungsumfrage der Sozialwissenschaften) gezogen wurde (GESIS 2018). Da wir nicht die Entwicklung der Einstellungen zu Immigranten untersuchen, sondern 
mögliche Determinanten identifizieren wollen, bezieht sich die Untersuchung auf das Jahr 2016, d.h. enthalten sind sowohl die Befragten der Auffrischungsstichprobe (Rekrutierungskohorte 2) als auch der vier Wellen für das entsprechende Jahr (dc-df). Damit fand die Datenerhebung knapp ein Jahr nach Beginn der ,Flüchtlingskrise“ statt. In unser Erhebungsjahr 2016 fallen auch Erfolge der AfD, die 2016 in insgesamt fünf Landesparlamente einziehen konnte und dabei in ihren Wahlkämpfen xenophobe und nativistische Positionen bediente. Aber auch die PEGIDA-Bewegung erfuhr bereits bundesweit Unterstützung in der Gesellschaft (Grimm 2015, S. 273), was als Anzeichen für einen sich verschärfenden, gesellschaftlichen Diskurs über Migration und Flüchtlinge gewertet werden kann. Somit bildet dieses Erhebungsjahr eine gute Ausgangslage, um migrationsskeptische Einstellungen näher zu beleuchten.

Das Panel besteht aus einem Kernmodul von GESIS (,Welcome Survey“) sowie soziodemografischen Variablen und einstellungsbezogenen Fragen. Ergänzt wird diese Kernstruktur um wellenspezifische Fragemodule, die extern eingereicht wurden (Kolb und Weyandt 2018). Insgesamt sind 2124 Befragte im Datensatz enthalten, von denen 1710 als aktive Panelisten gelten (Schaurer und Weyandt 2018, S. 6). Der „Welcome Survey (d12)“ beinhaltet ALLBUS-Items, die Einstellungen zu Flüchtlingen sowie soziodemografische Angaben über Alter, Geschlecht und Bildungsabschluss erheben. Die verwendeten Wellen (dc-df) enthalten unter anderem Fragen zu gruppenspezifischen Einstellungen in Bezug auf Minderheiten, Nationalstolz sowie Instrumente der Allgemeinen Bevölkerungsumfrage der Sozialwissenschaften (ALLBUS) zur Integration von Ausländern, der Einstellung gegenüber dem Islam und dem allgemeinen Vertrauen in andere Menschen ${ }^{6}$.

Unsere abhängige Variable „Einstellungen zu Flüchtlingen“ haben wir aus vier Items gebildet, welche Einstellungen zu Chancen bzw. Risiken durch die Zuwanderung von Flüchtlingen für die Bereiche „Sozialstaat“, „öffentliche Sicherheit““, „Zusammenleben in der Gesellschaft" und ,wirtschaftliche Lage“ auf einer 5-stufigen Skala erheben. Der Wert 3 steht dabei für eine neutrale Sichtweise (,weder Chancen noch Risiken“). 1 und 2 auf der Antwortskala zeigen an, dass eher Chancen wahrgenommen werden; bei Werten größer 3 überwiegt die Erwartung von Risiken für die angeführten Bereiche ${ }^{7}$. Mittels einer explorativen Faktorenanalyse wurde die mögliche Mehrdimensionalität der Items geprüft, wobei sich deutlich ein gemeinsamer Faktor zeigte. Diese wurde zudem mittels einer konfirmatorischen Faktorenanalyse (CFA) geprüft und auch hier kristallisierte sich die einfaktorielle Struktur als die beste Option heraus (Cronbachs Alpha Wert von 0,85).

Die unabhängige Variable Bildung wurde über den erreichten formalen Bildungsgrad einer Person gemessen, wobei drei Gruppen (hohes Bildungsniveau, mittleres Bildungsniveau und niedriges Bildungsniveau) gebildet wurden. Analog dazu wurden auf der Basis des bedarfsgewichteten Haushaltsnettoeinkommens drei Einkommensgruppen gebildet (hohes Einkommen, mittleres Einkommen und niedriges

\footnotetext{
${ }^{6}$ Eine Übersicht zu den verwendeten Items findet sich im Anhang in Tab. 1, Zusatzmaterial-Online.

7 Diese Items erfassen eine Bewertung und beziehen sich damit auf das, was in der sozialpsychologischen Einstellungsforschung als affektive Einstellungsdimension bezeichnet wird. Solche Bewertungen bilden sich auf Basis eines bestimmten Wissens über das Einstellungsobjekt (kognitive Einstellungsdimension).
} 
Einkommen). Generelles soziales Vertrauen als weitere unabhängige Variable wurde über die Zustimmung zu der Aussage „Im Allgemeinen kann man Menschen vertrauen“" gemessen, wobei höhere Werte auf der 4-stufigen Antwortskala für eine Zustimmung stehen. Einige Autoren haben in den letzten Jahren die Validität dieses Standarditems zur Messung von generellem Vertrauen in Zweifel gezogen (Nannestad 2008; Reeskens und Hooghe 2007). Die messtheoretische Kritik entzündete sich dabei unter anderem an der semantisch sehr vagen Formulierung, die den Befragten einen weiten Spielraum lasse, selbst zu deuten, auf welche Gruppe von Personen sich ihre Vertrauenseinschätzung beziehen solle (Frings 2010, S. 237). So gehen Beiträge zum sogenannten Vertrauensradiusproblem (Delhey et al. 2011; Reeskens 2013) davon aus, dass der Kreis der Menschen, den die Befragten im Kopf haben, unterschiedlich groß sein und zum Beispiel Freunde, Angehörige der eigenen Nation oder ähnliches einschließen kann, keinesfalls aber automatisch auf Menschen ganz allgemein bezogen sein muss, die bei einer validen Messung des generellen Vertrauens eigentlich die Bezugsobjekte sein müssten. Entsprechend gab es einige Vorstöße, eine differenziertere Messung von sozialem Vertrauen zu initiieren, die aus mehreren Items besteht und zwischen Ingroup und Outgroup Trust differenziert, wobei Outgroup Trust dem generellen Vertrauen in andere Menschen entspricht (Crepaz et al. 2014). Da eine solche differenzierte Messung von sozialem Vertrauen im GESIS-Panel aber nicht enthalten ist, bleibt nur die Verwendung des oben angesprochenen Standarditems. Mit diesem Item trotzdem zu arbeiten, erscheint uns vertretbar, weil die oben erwähnten messtheoretischen Artikel zum Vertrauensradiusproblem zumindest zeigen konnten, dass das Standardmessinstrument in wohlhabenden, westlichen Ländern noch vergleichsweise gut funktioniert (Delhey et al. 2011; van der Linden et al. 2017, S. 3). Der generelle Kontakt mit Flüchtlingen wurde über die Frage „Hatten Sie schon einmal direkten persönlichen Kontakt mit Flüchtlingen?“" abgebildet, wobei die Antwortmöglichkeit zwischen „Ja“ und „Nein“ bestand. Personen, die nicht auf die Frage geantwortet haben, wurden aus der Untersuchung ausgeschlossen.

Komplexer gestaltete sich die Entwicklung eines Messvorschlags für das Konstrukt Ethnozentrismus, was dem Umstand geschuldet ist, dass sich in der sozialwissenschaftlichen Forschung bislang keine einheitliche Konzeptualisierung und Operationalisierung von Ethnozentrismus durchsetzen konnte; vielfältige Definitionsund Operationalisierungsvorschläge existieren nebeneinander (Bizumic und Duckitt 2012). Propagiert werden einerseits Messkonzepte, die nur auf negative Haltungen gegenüber der Outgroup fokussiert sind und Ethnozentrismus über Vorurteile gegenüber anderen ethnischen Gruppen fassen, andererseits Konzeptualisierungen, die stattdessen eher auf die Ingroup und deren Überbewertung gegenüber der Outgroup ausgerichtet sind (Bizumic und Duckitt 2012, S. 889). Wir orientieren uns an einer dritten bereits validierten Messvariante, die Ethnozentrismus als ein zweidimensionales Konstrukt fasst und sowohl solche Einstellungsitems miteinbezieht, die die Idealisierung der Eigengruppe messen, als auch solche, die sich auf die Abwertung der Fremdgruppe beziehen (Schmidt und Heyder 2003; Herrmann 2001). Da Ethnozentrismus vor allem ein Differenzbegriff ist, der sich auf eine als „Wir“ verstandene politische Gemeinschaft bezieht und diese zugleich von einer nicht dazugehörigen Umwelt der „Anderen“ abgrenzt (Fuchs et al. 1993, S. 238), halten wir diesen Ope- 
rationalisierungsvorschlag für am besten geeignet. Zur Messung der Idealisierung der Eigengruppe verwenden wir in Anlehnung an Schmidt und Heyder daher das Item Nationalstolz, das die positive Haltung zum „Deutschsein“ und den Deutschen als relevante Eigengruppe misst. Aus theoretischer Perspektive ist Nationalstolz Teil des übergeordneten Ethnozentrismus, da hier die Eigengruppe definiert wird und die Zugehörigkeit zur Nation gleichzeitig als Abgrenzungskriterium zu anderen Gruppen fungiert (Rosar 2001, S. 30; Kende et al. 2018). Im Datensatz sind zwei Items enthalten, die Bezug zu Nationalstolz aufweisen: Zum einen ist dies die Frage ob man ,stolz ist Deutscher zu sein“. Dieses Item, das eine positive Haltung zur eigenen Nation und damit Patriotismus erhebt, stellt eine ,weichere“ Form des Nationalstolzes dar (denkbar ist hier, dass Befragte die Frage auf bestimmte Institutionen oder spezifische kulturelle Errungenschaften beziehen). Zum anderen gibt es die Frage, ob „Deutsch sein Teil der Persönlichkeit“" ist, was noch viel deutlicher auf eine starke positive Identifizierung mit der eigenen Nationalität als Eigengruppe abzielt. Beide Items sind 5-stufig, wobei höhere Werte für Nationalstolz stehen.

Zur Operationalisierung der Abwertung der Fremdgruppe haben wir erstens das im GESIS-Panel enthaltene ALLBUS-Instrument „Integration von Ausländern“ genutzt. Es enthält vier 7-stufige Items, die die Zustimmung respektive Ablehnung zu folgenden Aussagen erheben: „Ausländer sollten Ihre Ehepartner unter den eigenen Landsleuten wählen“, ,,wenn Arbeitsplätze knapp werden, sollte man die in Deutschland lebenden Ausländer zurück in ihre Länder schicken“, „Ausländer sollten sich der deutschen Lebensweise anpassen“ und ,politische Beteiligung von Ausländern sollte beschränkt werden“" (ZA/ZUMA 2014). Zustimmung zu diesen Items zeugt von einem Denken in Wir-vs. die Anderen-Kategorien und einer geringschätzigen und ablehnenden Haltung gegenüber Ausländern. Die Güte des Konstrukts ist bereits getestet worden (Blank und Schwarzer 1994) und auch unsere Faktorenanalyse bestätigt die Zugehörigkeit der vier Statements zu einem gemeinsamen latenten Faktor „Ausländerfeindlichkeit“.

Zur Messung der Abwertung der Fremdgruppe beziehen Heyder und Schmidt (2003) wie auch Herrmann (2001, S. 64) außerdem „Antisemitismus“ mit ein und berücksichtigen damit den Umstand, dass Abgrenzung oft auch über religiöse Merkmale erfolgen kann. Dem stimmen wir zu, schlagen aber stattdessen vor, Fremdgruppenabwertung über negative Einstellungen zum Islam und nicht zum Judentum zu operationalisieren. Zwar ist auch Antisemitismus in Deutschland kein Randphänomen, sondern nach wie vor in der deutschen Gesellschaft in nennenswertem Ausmaß verbreitet (Unabhängiger Expertenkreis Antisemitismus 2017, S. 13). Es sind aber der Islam und die Gruppe der Muslime, die derzeit als bedrohlich wahrgenommene Fremdgruppe in Verbindung mit gruppenbezogenen Vorurteilen deutlich stärker im Zentrum der öffentlichen Auseinandersetzung in Deutschland wie den meisten anderen nichtmuslimischen europäischen Ländern stehen (Pickel und Pickel 2019, S. 299; Pickel und Yendell 2016; Savekoul et al. 2011). Die gewachsene Bedeutung der Fremdgruppe „Muslime“ und die in den sich radikalisierenden öffentlichen Diskursen immer wieder beschworene Bedrohung der eigenen Identität durch eine vermeintliche „Islamisierung des Abendlands“, haben mittlerweile auch einen Widerhall in der Messtheorie gefunden. Klassische Skalen zur Erhebung von Eth- 
nozentrismus sind um Messinstrumente zur Erhebung von Islamophobie ergänzt worden (Pickel und Yendell 2016, S. 283).

Diesem Umstand Rechnung tragend, beziehen wir das Konstrukt „Islamophobie“ als weitere Messkomponente mit ein, welches ebenfalls durch ein ALLBUS-Instrument im Datensatz repräsentiert ist (Breyer und Danner 2015). Das Instrument umfasst insgesamt sechs Fragen, die von einer generellen Bewertung des Islam über die Forderung nach Einschränkung der Glaubenspraxis und Beobachtung durch den Staat bis hin zu stereotypen Zuschreibungen (, unter Muslimen befinden sich viele Fanatiker") reichen. Alle Items sind 7-stufig und wurden so umcodiert, dass hohe Werte eine ablehnende Einstellung repräsentieren. In Anlehnung an Heyder und Schmidt (2003, S. 189) wurde im Folgenden getestet, inwieweit die Indikatoren zu Ausländerfeindlichkeit, Nationalismus und Islamophobie voneinander getrennte Konstrukte darstellen oder ob diese einem gemeinsamen, latenten Faktor „Ethnozentrismus“ zuzuordnen sind. Zunächst wurden explorative Faktorenanalysen durchgeführt, um die möglichen latenten Konstrukte zu extrahieren. Aus der Hauptachsenanalyse konnten drei Faktoren extrahiert werden (das Kaiser-Meyer-Olkin-Kriterium [KMO] beträgt 0,84; zudem wurden die Faktoren varimax rotiert). Mittels Strukturgleichungsmodellen (SEM) wurde eine konfirmatorische Faktorenanalyse (CFA) Modell berechnet.

Aufgrund der hohen Anzahl an fehlenden Werten bei den angeführten Variablen (zwischen 670 und 700 Fälle) wurden die Modelle mit der Full-InformationMaximum-Likelihood-Methode (FIML) geschätzt, da sich diese im Gegensatz zu listenweisem Fallausschluss, paarweisem Ausschluss oder Imputation als effizientere Schätzmethode darstellt ${ }^{8}$ (Aichholzer 2017, S. 115). Das geschätzte Modell mit „Ethnozentrismus“ als Faktor höherer Ordnung, welches die aus der Theorie hergeleiteten Dimensionen „Nationalstolz“, „Ausländerfeindlichkeit“ und „Islamophobie“ als Subdimensionen enthält (Abb. 1), zeigte die beste Anpassung mit folgenden FitMaßen: $\chi 2$ (d.f.) 230.614 (41); RMSEA 0,056; CFI 0,972.

Weitere Tests ergaben zudem, dass Islamophobie sehr stark mit Ausländerfeindlichkeit (Kovarianz 0,916) assoziiert ist, was nicht verwundert, wenn man diese als spezifische Form der Fremdenfeindlichkeit begreift. Sowohl aus theoretischer als

\footnotetext{
8 Als Voraussetzung für den Schätzer wird jedoch eine multivariate Normalverteilung im Modell postuliert, die in der Analyse nach Tests auf multivariate Normalverteilung nicht gegeben ist. Dabei führt die Verletzung der Annahme zu verzerrten $\chi 2$-Werten und Standardfehlern der Parameter sowie verzerrten FitWerten. „Die Folge ist, dass auch ein im Grunde korrekt spezifiziertes Modell im Fall nicht normalverteilter Variablen eher abgelehnt wird, d.h. die globale Nullhypothese des Modells wird häufiger zurückgewiesen, obwohl sie in Wirklichkeit wahr ist, und die Modellparameter werden häufiger als ,signifikant " angesehen (Fehler 1. Art)“ (Aichholzer 2017, S. 114). Allerdings verweist Arzheimer (2016, S. 59) darauf, dass Maximum Likelihood-Verfahren bei ausreichend hoher Fallzahl trotzdem relativ robust gegenüber der Verletzung der Normalverteilung sind. Ory und Mokhtarian (2010, S. 442) hingegen warnen vor der Verwendung der ML-Schätzer, die tendenziell günstigere Ergebnisse lieferten, als dies tatsächlich der Fall sei. Um dem Problem Rechnung zu tragen, wurden die Modelle zudem mit dem Asymptotically Distribution Free (ADF) Schätzer (Arzheimer 2016, S. 59; Finney und Distefano 2006, S. 278; StataCorp 2017, S. 45), welcher allerdings eine hinreichend große Zahl an Fällen voraussetzt, sowie der von Aichholzer (2017, S. 114) empfohlenen Satorra-Bentler-Schätzung berechnet (Finney und Distefano 2006, S. 289). Da sich die Ergebnisse mit alternativen Schätzern inhaltlich nicht unterschieden, werden diese hier nicht weiter dargestellt.
} 


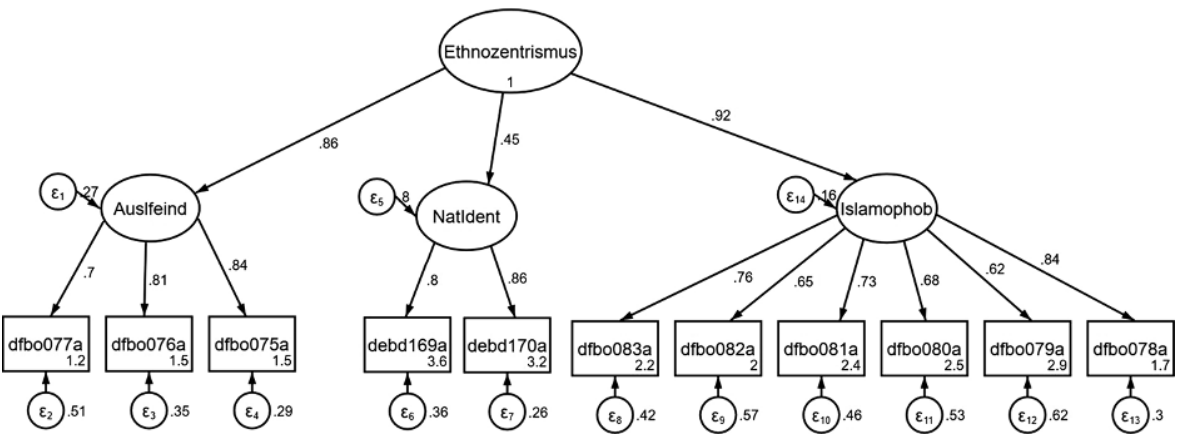

Abb. 1 CFA Ethnozentrismus. Quelle: GESIS (2018), eigene Berechnungen; Auslfeind Ausländerfeindlichkeit, NatIdent nationale Identität, Islamophob Islamophobie

auch aus empirischer Perspektive erscheint es somit unabdingbar, diese Dimension in die Analysen einzubeziehen.

Für die Analyse haben wir uns für ein lineares Strukturgleichungsmodell entschieden. Der Vorteil gegenüber einer linearen Regression liegt in der Möglichkeit, die Messung der latenten Konstrukte direkt in Verbindung mit dem Regressionsverfahren zu bringen (Aichholzer 2017, S. 7). Die Analysen wurden schrittweise aufgebaut, um einen Eindruck von den verschiedenen Effekten sowie deren Veränderung zu erhalten. Die Effekte der angeführten unabhängigen Variablen wurden unter Kontrolle von Alter (zentriert), Geschlecht (dichotom) und der Links-RechtsSelbsteinstufung (zentriert) geschätzt.

\section{Empirische Ergebnisse}

Einen ersten Eindruck bezüglich der Einstellungen zu Flüchtlingen vermitteln die folgenden deskriptiven Ergebnisse zur Verteilung der Mittelwerte, die wir zusätzlich nach den sozioökonomischen Merkmalen Bildung und Einkommen aufgeschlüsselt

Abb. 2 Chancen- und Risikoerwartung nach Einkommensgruppen. Quelle: GESIS (2018); eigene Darstellung

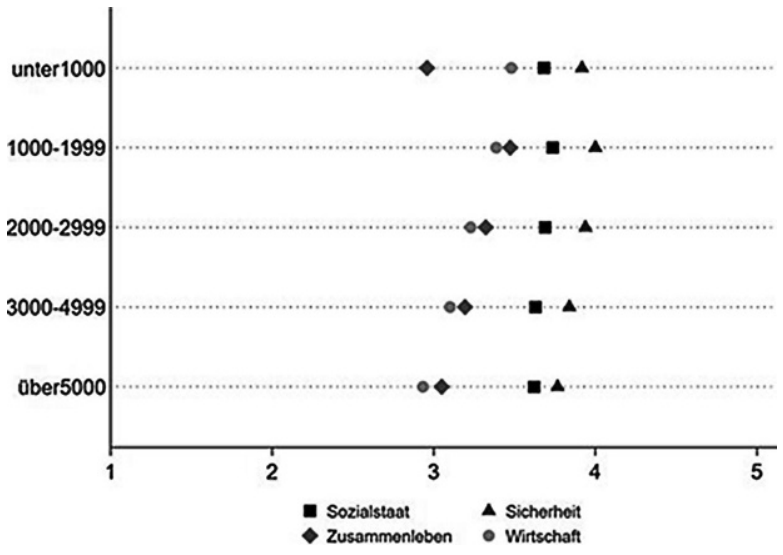


Abb. 3 Chancen- und Risikoerwartung nach Bildungsgruppen. Quelle: GESIS (2018); eigene Darstellung

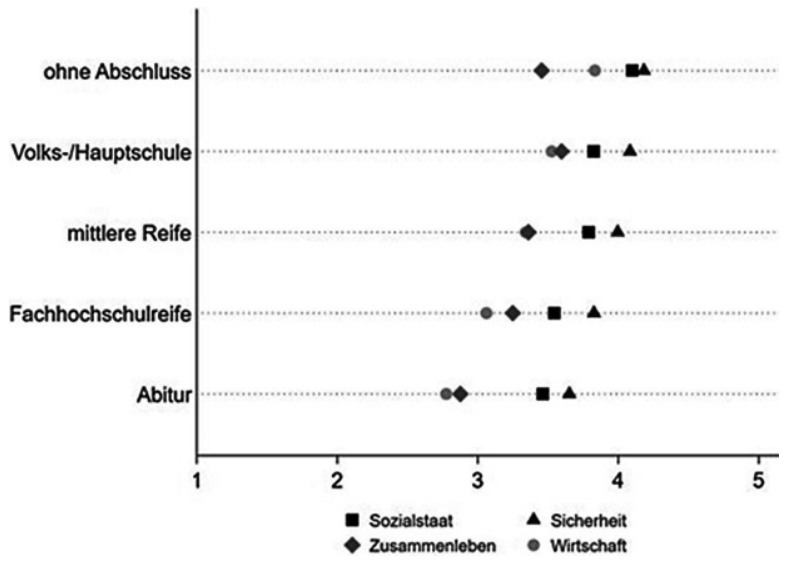

haben (Abb. 2). Befragte aller Einkommensgruppen bewerten demnach die Zuwanderung von Flüchtlingen, insbesondere in Bezug auf Fragen der Sicherheit und des Sozialstaats, eher als ein Risiko. Etwas optimistischer ist über alle Einkommensgruppen hinweg die Beurteilung der Auswirkungen auf die Wirtschaft und das Zusammenleben.

Auffällig ist, dass Bezieher sehr niedriger Einkommen (unter $1000 €$ ) eine neutrale Haltung hinsichtlich der Auswirkungen auf das Zusammenleben einnehmen und auch etwas weniger pessimistisch sind als die Befragten der Einkommensgruppe zwischen 1000 und 1999€, was Folgen für die Sicherheit und den Sozialstaat anbelangt. Die Einschätzung der ökonomischen Folgen der Flüchtlingszuwanderung fällt mit höherem Einkommen tendenziell positiver aus, die Unterschiede sind aber relativ gering.

Die Mittelwerte nach Bildungsgrad (Abb. 3) zeigen ähnliche Befunde. Besorgt sind die Befragten offenbar besonders hinsichtlich der Folgen von Flüchtlingszuwanderung für die Sicherheit und den Sozialstaat. Mit steigendem Bildungsabschluss sinken die Risikoerwartungen leicht. Personen mit Abitur zeigen im Mittel eine neutrale bis leicht optimistische Sicht auf die Folgen der Zuwanderung von Flüchtlingen für die Bereiche „Zusammenleben“ und „Wirtschaft“, während für den „Sozialstaat“ und die „Sicherheit“ auch hier eher Risiken erwartet werden. Insgesamt zeigen die deskriptiven Analysen, dass pessimistische Bewertungen eher überwiegen. Besonders für die Bereiche Sicherheit und Sozialstaat werden Nachteile erwartet und zwar - mit gewissen Abstufungen - über alle Einkommens- und Bildungsgruppen hinweg, sodass die Vermutung naheliegt, dass sozioökonomische Determinanten zwar einen gewissen, aber nicht besonders starken Effekt auf die Bewertung des Flüchtlingszuzugs haben. Dies zeigen auch die Ergebnisse unseres ersten Analysemodells (Modell $1^{9}$, Tab. 2, Zusatzmaterial-Online), das als unabhängige Variablen die sozioökonomischen Determinanten Bildung und Einkommen sowie Links-RechtsSelbsteinschätzung, Alter und Geschlecht als Kontrollvariablen beinhaltet. Einzig ein höheres Bildungsniveau zeigt einen überzufälligen $(p<0,001)$ Effekt auf die Be-

\footnotetext{
9 Fit-Maße: $\chi 2$ (d.f.) 62.256 (23); RMSEA 0,029; CFI 0,973.
} 
Abb. 4 Soziales Vertrauen und Erwartung von Flüchtlingszuwanderung. Quelle: GESIS (2018); eigene Berechnung; Full-Information-MaximumLikelihood mit standardisierten Koeffizienten; *** $p<0,001$, $* * p<0,01, * p<0,05$

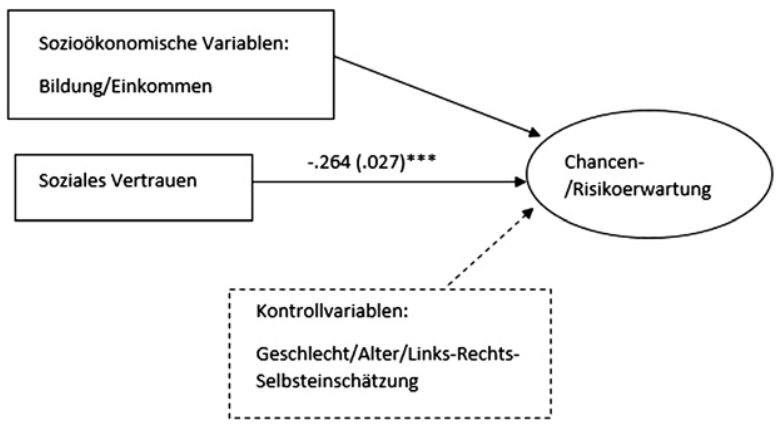

wertung von Zuwanderung. Für Menschen mit höherer Bildung stehen demnach eher Chancen von Zuwanderung im Vordergrund. Hingegen zeigen sich keine signifikanten Effekte für ein niedriges Bildungsniveau und Einkommen. Wie bereits auf Basis der deskriptiven Analyse vermutet, stützen diese Ergebnisse die Annahme, dass der soziökonomische Erklärungsansatz hier nur bedingt greift, eine Einschätzung, zu der auch Pickel und Pickel (2019) kommen. Die Links-Rechts-Selbsteinschätzung hingegen fällt hoch signifikant aus, was die Ergebnisse von Esses et al. (2017) bestätigt. Auch das Alter der Befragten scheint einen Einfluss zu haben. Menschen, die sich selbst eher rechts im politischen Raum verorten und Ältere sind demnach eher dazu geneigt, Risiken in der Zuwanderung zu sehen (Abb. 4).

In Modell $2^{10}$ wurde zusätzlich das soziale Vertrauen aufgenommen. Hier zeigt sich der in $H 2$ vermutete positive Effekt $(p<0,001)$. Personen mit höherem sozialen Vertrauen neigen dazu, mit der Flüchtlingszuwanderung mehr Chancen als Risiken zu verbinden. Die Ergebnisse für die sozioökonomischen Einflussfaktoren und die Kontrollvariablen bleiben im Vergleich zu Modell 1 stabil. Damit kann $H 2$ vorläufig als bestätigt gelten. Das ist insofern ein interessanter Befund, weil sich vermuten lässt, dass die Effekte des sozialen Vertrauens, aufgrund der Operationalisierung über das Standardvertrauensitem, eher noch zu konservativ ausfallen. Wie bereits erläutert wurde, lässt diese Art der Erhebung von sozialem Vertrauen den Vertrauensradius im Unklaren. Das heißt, es lässt sich nicht ermitteln, ob wirklich generelles Vertrauen in andere Menschen ganz allgemein, unabhängig von ethnischen, religiösen oder anderen Merkmalen der Distinktion, oder doch eher Ingroup trust erhoben wird. Gleichzeitig ist anzunehmen, dass Ingroup trust durchaus auch negativ auf Haltungen zur Einwanderung wirken könnte und nur generelles Vertrauen zu anderen Menschen ganz allgemein zu einer optimistischeren Einschätzung führt.

$\mathrm{Um} H 3 \mathrm{zu}$ überprüfen, haben wir in einem nächsten Analyseschritt Ethnozentrismus als weitere unabhängige Variable miteinbezogen (Modell 3) ${ }^{11}$. Verglichen mit den anderen Determinanten zeigt Ethnozentrismus den stärksten Effekt, womit sich $H 3$ bestätigt. Das Ergebnis zeigt deutlich, dass Menschen mit ethnozentrischen Haltungen mehr Risiken als Chancen mit dem Flüchtlingszuzug für die Gesellschaft verbinden $(p<0,001)$. Dieser Befund deckt sich mit bereits vorliegenden empiri-

10 Fit-Maße: $\chi 2$ (d.f.) 65.239 (26); RMSEA 0,027; CFI 0,975.

11 Fit-Maße: $\chi 2$ (d.f.) 721.496 (190); RMSEA 0,037; CFI 0,944. 
Abb. 5 Direkte und indirekte Effekte des sozialen Vertrauens und Erwartung von Flüchtlingszuwanderung. Quelle: GESIS (2018); eigene Berechnung; Full-Information-MaximumLikelihood mit standardisierten Koeffizienten; Effektzerlegung soziales Vertrauen: indirekt $\beta^{3 *}$ $\beta^{1}=-0,205(0,023)^{* * *}$; total: $\beta^{2}+\beta^{3 *} \beta^{1}=-0,253(0,03)^{* * *}$; $* * * p<0,001, * * p<0,01$, $* p<0,05 ; \beta^{1-3}$ (Koeffizienten aus der Berechnung zwischen den entsprechenden Variablen)

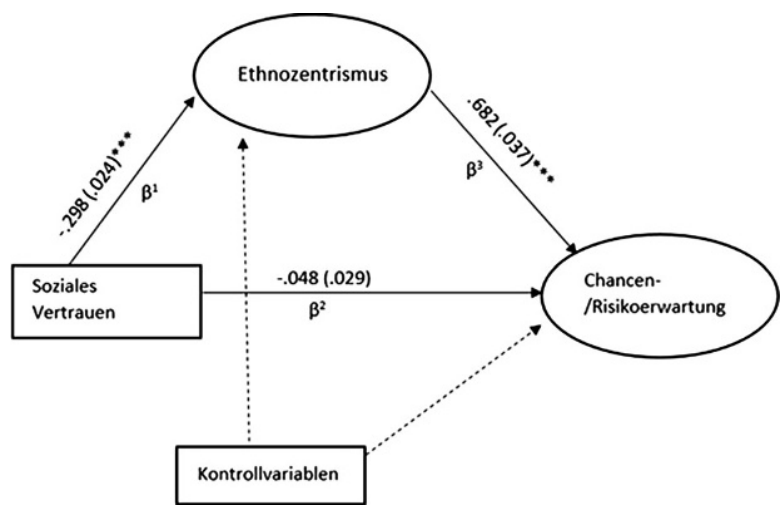

schen Analysen, die bestätigen, dass das Denken in Gruppenstereotypen einen starken Effekt auf Einstellungen zur Zuwanderung hat (Pickel und Pickel 2019, S. 292). Interessant ist zudem, dass soziales Vertrauen in Modell 3 insignifikant wird und damit an Erklärungskraft verliert. Auch die Effekte der sozioökonomischen Variablen sowie der Kontrollvariablen fallen jetzt insignifikant aus. Das führt zur Vermutung, dass soziales Vertrauen möglicherweise keinen direkten Effekt auf die Bewertung der Aufnahme von Flüchtlingen ausübt, sondern möglicherweise auch ethnozentrischen Einstellungen kausal vorgelagert sein könnte. Deswegen sind wir in unseren Analysen einen Schritt über die eingangs formulierten Hypothesen hinausgegangen und haben auch diese Möglichkeit eines indirekten Effekts ergänzend mittels Strukturgleichungsmodellen empirisch getestet. Sollte sich diese Annahme eines indirekten Effekts bestätigen, wäre dies ein wichtiger Anknüpfungspunkt für weitere Forschungsarbeiten und ein weiteres Argument dafür, die Rolle des sozialen Vertrauens stärker zu berücksichtigen.

Die empirische Analyse (Modell 512; Tab. 3, Zusatzmaterial-Online) zeigt sehr deutlich, dass Personen mit höherem sozialen Vertrauen signifikant $(p<0,001)$ weniger dazu neigen, ethnozentrische Einstellungen zu zeigen. Ebenso zeigen die Befunde (Abb. 5), dass gebildetere Menschen $(p<0,001)$ und Menschen mit einem höheren Einkommen $(p<0,001)$ weniger dazu neigen, ethnozentrische Einstellungen zu zeigen. Auch die subjektive Verortung als eher rechts im ideologischen Spektrum hat einen signifikanten Effekt auf Ethnozentrismus. Unser schrittweises Vorgehen bei der empirischen Analyse hat demnach zu dem Ergebnis geführt, dass generelles soziales Vertrauen offenbar weniger direkt, sondern eher vermittelt über ethnozentrische Haltungen auf die Beurteilung von Chancen und Risiken der Aufnahme von Flüchtlingen in Deutschland führt.

Zur Überprüfung von $H 4$ wurde in Modell $4^{13}$ (Abb. 6) der generelle Kontakt zu Flüchtlingen als weitere Determinante aufgenommen. Dahinter liegt die Vermutung, dass Kontakt einen positiven Effekt auf die Wahrnehmung von Flüchtlingen und somit auch auf die Chancen/Risikobewertung haben müsste. Andererseits erfasst das

12 Fit-Maße: $\chi 2$ (d.f.) 735.276 (203); RMSEA 0,036; CFI 0,443.

13 Fit-Maße: $\chi 2$ (d.f.) 735.276 (203); RMSEA 0,036; CFI 0,944. 
Abb. 6 Ergebnisse aus dem Modell 4. Quelle: GESIS (2018); eigene Berechnung; Full-Information-Maximum-Likelihood mit standardisierten Koeffizienten; $* * * p<0,001, * * p<0,01$, $* p<0,05$

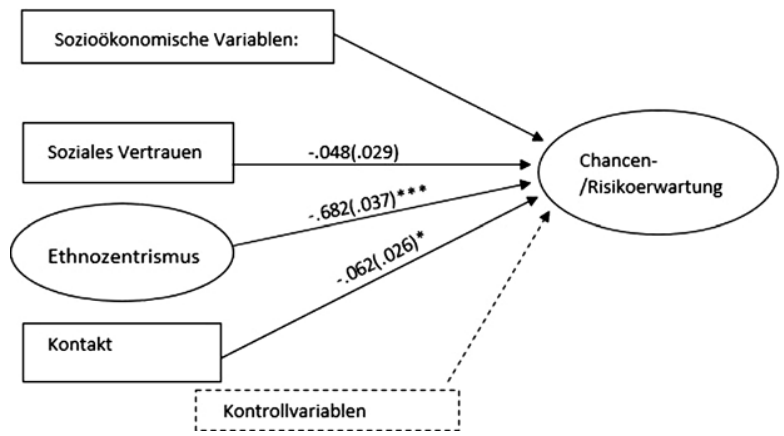

zur Verfügung stehende Item weder die Art noch die Intensität des Kontakts. Entsprechend könnte sich durchaus auch ein gegenteiliger Effekt zeigen, weil Kontakt auch zu negativen Erfahrungen mit Flüchtlingen führen kann. Interessanterweise bestätigt sich aber die vermutete positive Wirkung von Kontakt. Menschen, die in irgendeiner Form Kontakt zu Geflüchteten hatten, bewerten auch insgesamt den Zuzug für die Gesellschaft eher positiv $(p<0,05)$.

\section{Diskussion}

Positive Einstellungen auf Seiten der Mitglieder der Aufnahmegesellschaft scheinen eine wesentliche politisch-kulturelle Grundlage für gelingende Integration und Inklusion von Flüchtlingen zu sein. Wie der Zuzug einer größeren Anzahl von Flüchtlingen nach Deutschland im Jahr 2015 langfristig verkraftet wird und welche Auswirkungen er auf das gesellschaftliche Klima in Deutschland und die zukünftige Ausrichtung der deutschen Flüchtlingspolitik hat, scheint wesentlich von diesen Einstellungen abzuhängen. Vor diesem Hintergrund hat unser Beitrag beleuchtet, wie es mit den Einstellungen der Deutschen zur Integration von Flüchtlingen aussieht und welche Einstellungen diese Haltung bedingen.

Die verwendeten GESIS-Daten aus dem Jahr 2016 zeigen, dass die Wahrnehmung von Risiken unter den Deutschen stärker ausgeprägt ist als die Wahrnehmung von Chancen, ein Befund, der sich mit den Ergebnissen von Pickel und Pickel (2019, S. 289) deckt. Bestätigen lässt sich auch, dass es vor allem Sorgen hinsichtlich der Auswirkungen auf die Sicherheit sind, die die Deutschen umtreiben. Dahinter scheint die Angst zu stehen, dass durch die Aufnahme von Flüchtlingen aus überwiegend muslimisch geprägten Ländern die Gefahr eines islamistischen Terrorismus nach Deutschland importiert wird (Pickel und Pickel 2019, S. 292). Zudem befürchten die Deutschen offenbar vor allem, dass die aufgenommenen Flüchtlinge die sozialen Sicherungssysteme unter dem Strich mehr belasten als entlasten. Interessant ist, dass diese Einschätzung in allen Einkommensgruppen vertreten wird: Es sind nicht nur Menschen mit geringerem Einkommen, die Flüchtlinge eher als Belastung für den Sozialstaat wahrnehmen, sondern diese Einschätzung wird auch von Menschen in mittleren Einkommenslagen und von den Beziehern hoher Einkommen geteilt. Nur Menschen mit höherer Bildung sehen die Flüchtlingszuwanderung offenbar diffe- 
renzierter und optimistischer, was sich auch in anderen empirischen Studien schon gezeigt hat (Hainmueller und Hopkins 2014, S. 228; Schweitzer et al. 2005, S. 171). Hinsichtlich der untersuchten kulturellen Erklärungsvariablen erweisen sich vor allem ethnozentrische Haltungen als sehr erklärungsstark. Um den Effekt von Ethnozentrismus zu messen, haben wir in Anknüpfung an entsprechende messtheoretische Vorschläge im Forschungsstand zu Ethnozentrismus ein Messinstrument entwickelt und empirisch getestet, welches „Nationalstolz“, ,Ausländerfeindlichkeit“ und „Islamophobie“ als Subdimensionen enthält und damit die In- und Outputdistinktion über die nationale Zugehörigkeit um eine religiöse Komponente erweitert. Möglicherweise ist es auch hier in erster Linie die pauschale Verbindung von Geflüchteten mit ,dem Islam“ und eine generell ablehnende und skeptische Haltung gegenüber dem Islam, die für den starken Effekt des Ethnozentrismus verantwortlich ist (Pickel und Pickel 2019, S. 295; Pickel und Yendell 2016). Sinnvoll wäre es, die Validität dieser Ethnozentrismusmessung in weiteren empirischen Analysen genauer zu testen.

Auch soziales Vertrauen hat den vermuteten positiven Einfluss auf die Wahrnehmung von Chancen und Risiken der Zuwanderung. Damit bestätigen unsere Ergebnisse die Befunde anderer empirischer Studien, denen zufolge Menschen mit einem hohem Maß an sozialem Vertrauen offenbar über einen größeren Vertrauensradius verfügen, der auch Flüchtlinge mit einem anderen ethnischen oder religiösen Hintergrund mit einschließt (van der Linden et al. 2017, S. 9). Sinnvoll wäre es, diese Ergebnisse auf Basis alternativer Messinstrumente zur Vertrauensmessung zu validieren, die diesen Vertrauensradius explizit miteinbeziehen (Crepaz et al. 2014; Reeskens 2013). Dies böte interessante Ansatzpunkte für differenziertere Analysen zu den Vertrauenseffekten, insbesondere zu den zu vermutenden positiven Effekten von Outgroup Trust auf die Einstellungen zu Immigranten. Weiterer Forschungsbedarf besteht auch hinsichtlich der Frage, ob der Effekt des sozialen Vertrauens ein direkter Effekt ist oder eher vermittelt über Ethnozentrismus wirkt, worauf unsere Analysen Hinweise liefern. Folgt man der in der Forschung weit verbreiteten Perspektive, wonach Vertrauen eine früh in der Kindheit und Jugend erworbene persönliche Prädisposition ist, Menschen ganz generell eher zu vertrauen, und zwar unabhängig von Distinktionsmerkmalen wie Religionszugehörigkeit, ethnischer Zugehörigkeit oder Herkunft, dann wäre es nämlich auch plausibel anzunehmen, dass sich dieses generelle Vertrauen zuerst ausbildet und die im Sozialisationsverlauf möglicherweise später ausgeprägte Neigung zu ethnozentrischem Denken determiniert.

Kontakt mit Flüchtlingen hat ebenso den vermuteten positiven Effekt. Menschen, die in irgendeiner Form Kontakt zu Flüchtlingen hatten, bewerten die Zuwanderung eher als gesellschaftliche Chance. Ähnliches zeigen auch Gerhards et al. (2016), die herausstellen, dass die Offenheit und Hilfsbereitschaft von Personen, die bereits persönlichen Kontakt zu Flüchtlingen hatten, hoch ist, und zwar unabhängig davon, ob der Kontakt im beruflichen Kontext, im Alltag oder im Zuge von ehrenamtlichem Engagement zustande kam. Erwähnt sei allerdings kritisch, dass hier ein typisches Henne- Ei-Problem vorliegt. Nicht auszuschließen ist schließlich auf Basis unserer Studie die Möglichkeit einer umgekehrten Kausalität, wonach Menschen, die generell optimistischer gegenüber Zuwanderung eingestellt sind, auch öfter Kontakt zu 
Flüchtlingen suchen. Dieses Problem ließe sich nur auf Basis von weiterführenden Längsschnittstudien untersuchen.

Funding Open Access funding provided by Projekt DEAL.

Open Access Dieser Artikel wird unter der Creative Commons Namensnennung 4.0 International Lizenz veröffentlicht, welche die Nutzung, Vervielfältigung, Bearbeitung, Verbreitung und Wiedergabe in jeglichem Medium und Format erlaubt, sofern Sie den/die ursprünglichen Autor(en) und die Quelle ordnungsgemäß nennen, einen Link zur Creative Commons Lizenz beifügen und angeben, ob Änderungen vorgenommen wurden.

Die in diesem Artikel enthaltenen Bilder und sonstiges Drittmaterial unterliegen ebenfalls der genannten Creative Commons Lizenz, sofern sich aus der Abbildungslegende nichts anderes ergibt. Sofern das betreffende Material nicht unter der genannten Creative Commons Lizenz steht und die betreffende Handlung nicht nach gesetzlichen Vorschriften erlaubt ist, ist für die oben aufgeführten Weiterverwendungen des Materials die Einwilligung des jeweiligen Rechteinhabers einzuholen.

Weitere Details zur Lizenz entnehmen Sie bitte der Lizenzinformation auf http://creativecommons.org/ licenses/by/4.0/deed.de.

\section{Literatur}

Aichholzer, J. 2017. Einführung in lineare Strukturgleichungsmodelle mit Stata. Wiesbaden: Springer VS. https://doi.org/10.1007/978-3-658-16670-0.

Ajzen, I. 2001. Nature and operation of attitudes. Annual Review of Psychology 52:2758.

Ajzen, I., und M. Fishbein. 1980. Understanding attitudes and predicting social behavior. Englewood Cliffs: Prentice-Hall.

Ajzen, I., und M. Fishbein. 2005. The influence of attitudes on behavior. In The handbook of attitudes, Hrsg. D. Albarracín, B.T. Johnson, und M.P. Zanna, 173-221. Mahwah: Erlbaum.

Arzheimer, K. 2015. The AfD: finally a successful right-wing populist Eurosceptic party for Germany? West European Politics 38(3):535-556. https://doi.org/10.1080/01402382.2015.1004230.

Arzheimer, K. 2016. Strukturgleichungsmodelle. Eine anwendungsorientierte Einführung. Wiesbaden: Springer VS.

Arzheimer, K. 2017. Explaining electoral support for the radical right. In The oxford handbook of the radical right, Hrsg. J. Rydgren, 143-165. Oxford: University Press.

Banks, A.J. 2016. Are group cues necessary? How anger makes ethnocentrism among whites a stronger predictor of racial and immigration policy opinions. Political Behavior 38(3):635-657. https://doi. org/10.1007/s11109-016-9330-3.

Berg, J.A. 2015. Explaining attitudes toward immigrants and immigration policy: a review of the theoretical Literatur. Sociology Compass 9(1):23-34. https://doi.org/10.1111/soc4.12235.

Berning, C.C., und C. Ziller. 2017. Social trust and radical right-wing populist party preferences. Acta Politica 52(2):198-217. https://doi.org/10.1057/ap.2015.28.

Bertelsmann Stiftung (Hrsg.). 2017. Willkommenskultur im „Stresstest“. Einstellungen in der Bevölkerung 2017 und Entwicklungen und Trends seit 2011/2012. Ergebnisse einer repräsentativen Bevölkerungsumfrage. Gütersloh: Kantar Emnid.

Bizumic, B., und J. Duckitt. 2012. What is and is not ethnocentrism? A conceptual analysis and political implications. Political Psychology 33(6):887-909.

Blank, T., und S. Schwarzer. 1994. Ist die Gastarbeiterskala noch zeitgemäß? Die Reformulierung einer ALLBUS-Skala. ZUMA Nachrichten 18(34):97-115.

Breyer, B., und D. Danner. 2015. Einstellung zum Islam (ALLBUS). Zusammenstellung sozialwissenschaftlicher Items und Skalen https://doi.org/10.6102/zis231.

Ceobanu, A.M., und X. Escandell. 2010. Comparative analyses of public attitudes toward immigrants and immigration using multinational survey data: a review of theories and research. Annual Review of Sociology 36:309-328. https://doi.org/10.1146/annurev.soc.012809.102651.

Citrin, J., und J. Sides. 2008. Immigration and the imagined community in Europe and the United States. Political Studies 56:35-56. 
Crepaz, M.M.L., J.T. Polk, R.S. Bakker, und S.P. Singh. 2014. Trust matters: the impact of ingroup and outgroup trust on nativism and civicness. Social Science Quarterly 85(2):938-959. https://doi.org/10. $1111 /$ ssqu. 12082 .

Czymara, C.S., und A.W. Schmidt-Catran. 2016. Wer ist in Deutschland willkommen? Eine Vignettenanalyse zur Akzeptanz von Einwanderung. Kölner Zeitschrift für Soziologie und Sozialpsychologie 68:193-227. https://doi.org/10.1007/s11577-016-0361-x.

Davidov, E., B. Meuleman, J. Billiet, und P. Schmidt. 2008. Values and support for immigration: a crosscountry comparison. European Sociological Review 24(5):583-599. https://doi.org/10.1093/esr/ jen020.

Decker, F. 2015. Alternative für Deutschland und Pegida. Die Ankunft des neuen Rechtspopulismus in der Bundesrepublik. In Rechtspopulismus und Rechtsextremismus in Europa. Die Herausforderung der Zivilgesellschaft durch alte Ideologien und neue Medien, Hrsg. B. Henningsen, K. Jakobsen, 75-90. Baden-Baden: Nomos.

Decker, F. 2016. Die „Alternative für Deutschland“ aus der vergleichenden Sicht der Parteienforschung. In Die Alternative für Deutschland Programmatik, Entwicklung und politische Verortung, Hrsg. A. Häusler, 7-23. Wiesbaden: Springer VS.

Delhey, J., K. Newton, und C. Welzel. 2011. How general is trust in "most people"? Solving the radius of trust problem. American Sociological Review 76(5):786-807. https://doi.org/10.1177/ 0003122411420817.

Eagly, A., und S. Chaiken. 1993. The psychology of attitudes. New York: Harcourt Brace.

Eisnecker, P., und J. Schupp. 2016. Flüchtlingszuwanderung: Mehrheit der Deutschen befürchtet negative Auswirkungen auf Wirtschaft und Gesellschaft. DIW Wochenbericht 8:158-164.

Esses, V.M., L.K. Hamilton, und D. Gaucher. 2017. The global refugee crisis: empirical evidence and policy implications for improving public attitudes and facilitating refugee resettlement. Social Issues and Policy Review 11(1):78-123. https://doi.org/10.1111/sipr.12028.

Fetzer, J.S. 2000. Public attitudes toward immigration in the United States, France, and Germany. Cambridge: University Press.

Fietkau, S. 2016. Einstellungen gegenüber Immigranten in Deutschland. Abstraktionsebenen und Erklärungsansätze. Dissertation. Mainz: Johannes Gutenberg-Universität Mainz.

Finney, S.J., und C. DiStefano. 2006. Non-normal and categorical data in structural equation modeling. In Structural equation modeling: a second course, Hrsg. G.R. Hancock, Mueller R.O. Greenwich, 439-492. CT: Information Age Publishing.

Freeman, G. P. 1995. Modes of Immigration Politics in Liberal Democratic States. International Migration Review 29(4):881. https://doi.org/10.2307/2547729.

Freitag, M., und C. Rapp. 2013. Intolerance toward immigrants in Switzerland: diminished threat through social contacts? Swiss Political Science Review 19(4):425-446. https://doi.org/10.1111/spsr.12049.

Frings, C. 2010. Soziales Vertrauen. Eine Integration der soziologischen und der ökonomischen Vertrauenstheorie. Wiesbaden: VS. https://doi.org/10.1007/978-3-531-92297-3.

Fuchs, D., J. Gerhards, und E. Roller. 1993. Wir und die anderen. Ethnozentrismus in den zwölf Ländern der europäischen Gemeinschaft. Kölner Zeitschrift für Soziologie und Sozialpsychologie 45:238-253.

Geiges, L., S. Marg, und F. Walter. 2015. Pegida. Die schmutzige Seite der Zivilgesellschaft? Bielefeld: transcript.

Gerhards, J., S. Hans, und J. Schupp. 2016. Einstellungen der BürgerInnen in Deutschland zur Aufnahme von Geflüchteten. DIW-Wochenbericht 83(21):467-473.

GESIS. 2018. GESIS Panel - Standard Edition. ZA5665 Datenfile Version 28.0.0. Köln: GESIS Datenarchiv. https://doi.org/10.4232/1.13210.

Gorodzeisky, A., und M. Semyonov. 2019. Unwelcome immigrants: sources of opposition to different immigrant groups among Europeans. Frontiers in Sociology https://doi.org/10.3389/fsoc.2019.00024.

Grimm, R. 2015. The rise of the German Eurosceptic party Alternative für Deutschland, between ordoliberal critique and popular anxiety. International Political Science Review 36(3):264-278.

Hainmueller, J., und M.J. Hiscox. 2007. Educated preferences: explaining attitudes toward immigration in europe. International Organization 61(2):399-442. https://doi.org/10.1017/S0020818307070142.

Hainmueller, J., und D.J. Hopkins. 2014. Public attitudes toward immigration. Annual Review of Political Science 17:225-249. https://doi.org/10.1146/annurev-polisci-102512-194818.

Hambauer, V., und A. Mays. 2018. Wer wählt die AfD? - Ein Vergleich der Sozialstruktur, politischen Einstellungen und Einstellungen zu Flüchtlingen zwischen AfD-WählerInnen und der WählerInnen anderer Parteien. Zeitschrift für Vergleichende Politikwissenschaft 12:133-154. https://doi.org/10.1007/ s12286-017-0369-2. 
Haubert, J., und E. Fussell. 2006. Explaining pro-immigrant sentiment in the U.S.: Social class, cosmopolitanism, and perceptions of immigrants. International Migration Review 40(3):489-507. https://doi. org/10.1111/j.1747-7379.2006.00033.x.

Herreros, F., und H. Criado. 2009. Social trust, social capital and perceptions of immigration. Political Studies 57(2):337-355. https://doi.org/10.1111/j.1467-9248.2008.00738.x.

Herrmann, A. 2001. Ursachen des Ethnozentrismus in Deutschland. Zwischen Gesellschaft und Individuum. Opladen: Leske + Budrich.

Heyder, A., und P. Schmidt. 2003. Authoritarianism and ethnocentrism in east and west Germany-does the system matter? In Germans or foreigners? Attitudes toward ethnic minorities in post-reunification Germany, Hrsg. R. Alba, P. Schmidt, und M. Wasmer, 187-210. New York: Palgrave MacMillan.

Hooghe, M., C. Meeusen, und E. Quintelier. 2013. The impact of education and intergroup friendship on the development of ethnocentrism: a latent growth curve model analysis of a five-year panel study among Belgian late adolescents. European sociological review 29(6):1109-1121. https://doi.org/10. 1093/esr/jcs086.

Houston, D.A., und R.H. Fazio. 1989. Biased processing as a function of attitude accessibility: making objective judgements subjectively. Social cognition 7(1):51-66. https://doi.org/10.1521/soco.1989.7. 1.51 .

Inglehart, R. 1970. Cognitive mobilization and European identity. Comparative Politics 3(1):45-70.

Kende, A., M. Hadarics, und Z.P. Szabó. 2018. Inglorious glorification and attachment: National and European identities as predictors of anti- and pro-immigrant attitudes. British Journal of Social Psychology 58(3):569-590. https://doi.org/10.1111/bjso.12280.

Kinder, D.R., und C.D. Kam. 2009. Us against them: ethnocentric foundations of American opinion. Chicago: University Press.

Kolb, J.P., und K. Weyandt. 2018. Gesis panel data manual.

Lahav, G. 2004. Public opinion toward immigration in the European Union: does it matter? Comparative Political Studies 37(10):1151-1183.

Lengfeld, H. 2017. Die „Alternative für Deutschland“: eine Partei für Modernisierungsverlierer? Kölner Zeitschrift für Soziologie und Sozialpsychologie 69:209-232.

Lengfeld, H., und C. Dilger. 2018. Kulturelle und ökonomische Bedrohung. Eine Analyse der Ursachen der Parteiidentifikation mit der „Alternative für Deutschland“ mit dem Sozioökonomischen Panel 2016. Zeitschrift für Soziologie 47(3):181-199. https://doi.org/10.1007/s11577-017-0446-1.

Lewandowsky, M., H. Giebler, und A. Wagner. 2016. Rechtspopulismus in Deutschland. Eine empirische Einordnung der Parteien zur Bundestagswahl 2013 unter besonderer Berücksichtigung der AfD. Politische Vierteljahresschrift 57(2):247-275. https://doi.org/10.5771/0032-3470-2016-2-247.

Van der Linden, M., M. Hooghe, T. de Vroome, und C. Van Laar. 2017. Extending trust to immigrants: generalized trust, cross-group friendships and anti-immigrant sentiments in 21 European societies. PLos ONE https://doi.org/10.1371/journal.pone.0177369.

Louis, W.R., J.M. Duck, D.J. Terry, R.A. Schuller, und R.N. Lalonde. 2007. Why do citizens want to keep refugees out? Threats, fairness and hostile norms in the treatment of asylum seekers. European Journal of Social Psychology 37(1):53-73. https://doi.org/10.1002/ejsp.329.

Malchov-Møller, N., J.R. Munch, S. Schroll, und J.R. Skaksen. 2009. Explaining cross-country differences in attitudes towards immigration in the EU-15. Social Indicators Research 91(3):371-390. https://doi. org/10.1007/s11205-008-9341-5.

Marfouk, A. 2018. I am neither racist nor xenophobic, but: dissecting European attitudes towards a ban on Muslims' immigration. Ethnic and Racial Studies 42:1747-1765. https://doi.org/10.1080/01419870. 2018.1519585.

Margaryan, S., A. Paul, und T. Siedler. 2018. Does education affect attitudes towards immigration? Evidence from Germany. IZA discussion paper no. 11980.

Markus, H.R., und R.B. Zajonc. 1985. The cognitive perspective in social psychology. In Handbook of Social Psychology, Bd. 1, Hrsg. L. Gardner, E. Aronson, 137-230. New York: Random House.

Mayda, A.M. 2004. Who is against immigration? A cross-country investigation of individual attitudes toward immigrants. IZA discussion paper no. 1115.

McLaren, L.M. 2003. Anti-immigrant prejudice in europe: contact, threat perception, and preferences for the exclusion of migrants. Social Forces 81(3):909-936.

Meuleman, B., E. Davidov, und J. Billiet. 2009. Changing Attitudes toward immigration in Europe, 2002-2007: a dynamic group conflict theory approach. Social Science Research 38(2):351-365. https://doi.org/10.1016/j.ssresearch.2008.09.006.

Mudde, C. 2017. The populist radical right. a reader. London, New York: Routledge. 
Mughan, A., und P. Paxton. 2006. Anti-immigrant sentiment, policy preferences and populist party voting in Australia. British Journal of Political Science 36(2):341-358.

Nannestad, P. 2008. What have we learned about generalized trust, if anything? Annual Review of Political Science 11(1):413-436. https://doi.org/10.1146/annurev.polisci.11.060606.135412.

Ory, David T., und Patricia L. Mokhtarian. 2010. The impact of non-normality, sample size and estimation technique on goodness-of-fit measures in structural equation modeling: evidence from ten empirical models of travel behavior. Quality \& Quantity 44(3):427-445. https://doi.org/10.1007/s11135-0089215-6.

Paxton, P. 2002. Social capital and democracy. An interdependent relationship. American Sociological Review 67(2):254-277. https://doi.org/10.2307/3088895.

Pettigrew, T.F., L.R. Tropp, U. Wagner, und O. Christ. 2011. Recent advances in intergroup contact theory. International Journal of Intercultural Relations 35(3):271-280. https://doi.org/10.1016/j.ijintrel. 2011.03.001.

Pickel, G., und S. Pickel. 2018. Migration als Gefahr für die politische Kultur? Kollektive Identitäten und Religionszugehörigkeit als Herausforderung demokratischer Gemeinschaften. Zeitschrift für Vergleichende Politikwissenschaft 12:297-320. https://doi.org/10.1007/s12286-018-0380-2.

Pickel, G., und S. Pickel. 2019. Der Flüchtling als Muslim - und unerwünschter Mitbürger? In Flucht und Migration in Europa, Politik und Religion. Neue Herausforderungen für Parteien, Kirchen und Religionsgemeinschaften, Hrsg. O. Hidalgo, G. Pickel, 279-322. Wiesbaden: VS.

Pickel, G., und A. Yendell. 2016. Islam als Bedrohung? Beschreibung und Erklärung von Einstellungen zum Islam im Ländervergleich. Zeitschrift für Vergleichende Politikwissenschaft 10:273-309.

Pickel, G., A. Röder, und A. Blätte. 2018. Migration und (demokratische) politische Kultur - ein dynamisches und polarisierendes Thema? Zeitschrift für Vergleichende Politikwissenschaft 12:1-7. https:// doi.org/10.1007/s12286-018-0382-0.

Putnam, R.D., R. Leonardi, und R.Y. Nonetti. 1993. Making democracy work: civic traditions in modern Italy. Princeton: Princeton University Press.

Reeskens, T. 2013. But who are those "most people" that Can be trusted? Evaluating the radius of trust across 29 European societies. Social Indicators Research 114(2):703-722. https://doi.org/10.1007/ s11205-012-0169-7.

Reeskens, T., und M. Hooghe. 2007. Cross-cultural measurement equivalence of generalized trust. Evidence from the European Social Survey (2002 and 2004). Social Indicators Research 85(3):515-532. https://doi.org/10.1007/s11205-007-9100-z.

Rippl, S., und C. Seipel. 2018. Modernisierungsverlierer, Cultural Backlash, Postdemokratie. Was erklärt rechtspopulistische Orientierungen? Kölner Zeitschrift für Soziologie und Sozialpsychologie 70(2):237-254. https://doi.org/10.1007/s11577-018-0522-1.

Rosar, U. 2001. Ethnozentrismus in Deutschland. Eine komparative Analyse 1980 bis 1996. Wiesbaden: Westdeutscher Verlag.

Rucht, D., P. Daphi, P. Kocyba, M. Neuber, J. Roose, F. Scholl, M. Sommer, W. Stuppert, und S. Zajak. 2015. Protestforschung am Limit. Eine soziologische Annäherung an Pegida. Berlin: Institut für Protest- und Bewegungsforschung.

Rustenbach, E. 2010. Sources of negative attitudes toward immigrants in Europe: a multi-level analysis. International Migration Review 44(1):53-77.

Rydgren, J. 2008. Immigration sceptics, xenophobes or racists? Radical right-wing voting in six West European countries. European Journal of Political Research 47(6):737-765. https://doi.org/10.1111/ j.1475-6765.2008.00784.x.

Savelkoul, M., P. Scheepers, J. Tolsma, und L. Hagendoorn. 2011. Anti-muslim attitudes in the Netherlands: tests of contradictory hypotheses derived from ethnic competition theory and intergroup contact theory. European Sociological Review 27(6):741-758. https://doi.org/10.1093/esr/jcq035.

Schammann, H. 2015. PEGIDA und die deutsche Migrationspolitik. Ein Beitrag zur Differenzierung des Opinion-Policy-Gaps in der Migrationsforschung. Zeitschrift für Politikwissenschaft 25(3):309-333. https://doi.org/10.5771/1430-6387-2015-3-309.

Schaurer, I., und K. Weyandt. 2018. GESIS panel technical report. Recruitment 2016 (wave d11 and d12).

Scheve, K.F., und M.J. Slaughter. 2001. Labor market competition and individual preferences over immigration policy. The Review of Economics and Statistics 83(1):133-145.

Schmidt, P., und S. Weick. 2017. Kontakte und die Wahrnehmung von Bedrohungen besonders wichtig für die Einschätzung von Migranten: Einstellungen der deutschen Bevölkerung zu Zuwanderern von 1980 bis 2016. Informationsdienst Soziale Indikatoren 57:1-7. https://doi.org/10.15464/isi.57.2017. $1-7$. 
Schmitt-Beck, R., J. van Deth, und A. Staudt. 2017. Die AFD nach der rechtspopulistischen Wende. Wählerunterstützung am Beispiel Baden-Württembergs. Zeitschrift für Politikwissenschaft 27(3):273-303. https://doi.org/10.1007/s41358-017-0104-1.

Schweitzer, R., S. Perkoulidis, S. Krome, C. Ludlow, und M. Ryan. 2005. Attitudes towards refugees: the dark side of prejudice in Australia. Australian Journal of Psychology 57(3):170-179. https://doi.org/ 10.1080/00049530500125199.

Semyonov, M., R. Raijman, A. Yom Tov, und P. Schmidt. 2004. Population size, perceived threat, and exclusion: a multiple-indicator analysis of attitudes toward foreigners in Germany. Social Science Research 33(4):681-701. https://doi.org/10.1016/j.ssresearch.2003.11.003.

Sides, J., und J. Citrin. 2007. European opinion about immigration: the role of identities, interests and information. British Journal of Political Science 37(3):477-504. https://doi.org/10.1017/ S0007123407000257.

StataCorp, L.L.C. 2017. Structural equation modeling. Reference Manual. Version 15. Texas: Stata Press.

SVR. 2018. Stabiles Klima in der Integrationsrepublik Deutschland. SVR-Integrationsbarometer 2018. Berlin: SVR.

Tajfel, H., J.C. Turner, W.G. Austin, und S. Worchel. 1979. An integrative theory of intergroup conflict. Organizational identity. In The social psychology of intergroup relations, Hrsg. W.G. Austin, S. Worchel, 56-65. Monterey: Brooks/Cole Pub. Co.

Unabhängiger Expertenkreis Antisemitismus. 2017. Antisemitismus in Deutschland - aktuelle Entwicklungen.

Valentino, N.A., T. Brader, und A.E. Jardina. 2013. Immigration opposition among U.S. Whites: general Ethnocentrism or media priming of attitudes about latinos? Political Psychology 34(2):149-166. https://doi.org/10.1111/j.1467-9221.2012.00928.x.

Weber, H. 2016. Mehr Zuwanderer - mehr Fremdenangst? Ein Überblick über den Forschungsstand und ein Erklärungsversuch aktueller Entwicklungen in Deutschland. Berliner Journal für Soziologie 25:397-428. https://doi.org/10.1007/s11609-016-0300-8.

Weins, C. 2004. Fremdenfeindliche Vorurteile in den Staaten der EU. Wiesbaden: VS.

Zamora-Kapoor, A., P. Kovincic, und C. Causey. 2013. Anti-foreigner sentiment: state of the art. Sociology Compass 7(4):303-314. https://doi.org/10.1111/soc4.12027.

Zentralarchiv für empirische Sozialforschung (ZA), und Zentrum für Umfragen, Methoden und Analysen (ZUMA) e. V.. 2014. Integration von Ausländern (ALLBUS). https://doi.org/10.6102/zis224. Zusammenstellung sozialwissenschaftlicher Items und Skalen.

Zmerli, S., und K. Newton. 2008. Social trust and attitudes towards democracy. Comparative Political Studies 72(4):706-724. https://doi.org/10.1093/poq/nfn054. 